\title{
THE MACKEY TOPOLOGY AS A MIXED TOPOLOGY
}

\author{
J. B. COOPER
}

ABSTRACT. A theorem on the coincidence of a mixed topology and the Mackey topology is given. The techniques of partitions of unity are used. As a corollary, a result of LeCam and Conway on the strict topology is obtained. Similar methods are applied to obtain a theorem of Collins and Dorroh.

Introduction. In recent years the space $C(S)$ of bounded, continuous complex-valued functions on a locally compact space $S$ with the strict topology has received a great deal of attention. In [5] we have shown that this topology was a special case of a mixed topology in the sense of the Polish school. Many of the results on the strict topology are, in fact, special cases of general results on mixed topologies and, when this is the case, it seems to us to be desirable to present this approach since it displays the strict topology as part of a larger scheme rather than as an isolated phenomenon.

In this note, we give an example of this process by considering the problem: When is the strict topology on $C(S)$ the Mackey topology? LeCam and Conway have shown that this is the case when $S$ is paracompact. In fact, there are a number of results of this type, that is, identifying mixed topologies with the Mackey topology (see Cooper [6], Stroyan [13]) and this suggests that there might be a general theorem of this type in the context of mixed topologies. In this note, we give such a theorem ( 11 below).

Although the result on $C(S)$ that we prove (12 below) is not new, it seemed to us worthwhile to give a proof of this type since it shows that the problem is mainly one in functional analysis and that measure theoretical techniques, for example, are unnecessary. In addition, we feel that the methods that we use are of some interest in themselves. We mention three points. It is known that the duality between sums and products in the category of locally convex spaces breaks down in the category of Banach spaces. We show how this duality can be recovered by using mixed topologies (5(i) and (ii)). The result 5(iii) can be regarded as an infinite dimensional version of Schur's theorem (if each $E_{\alpha}$ is one dimensional we obtain the classical Schur theorem-of course, we have used the latter in the proof of 5(iii)). Thirdly, we use the technique of partitions of unity which were introduced by De Wilde [16] for locally convex spaces. It is our opinion that this technique can be ex-

Received by the editors September 23, 1974.

AMS (MOS) subject classifications (1970). Primary 46A05, 46E 10.

Key words and phrases. Mixed topology, Mackey topology, the strict topology, Banach-Steinhaus property. 
tended to most of the interesting categories of functional analysis, and that it is particularly fruitful in applications to function spaces.

We take the opportunity to consider a similar result on $(C(S), \beta)$ for paracompact $S$, namely that its dual space is weakly sequentially complete (Collins and Dorroh [3]). We show that this can also be seen (in an apparently stronger form-but only apparently in view of the fact that $(C(S), \beta)$ is a Mackey space) as a special case of a general result on mixed topologies. The techniques are essentially the same as those used above.

Our methods can also be applied to other function spaces and can be used to give a version of the LeCam-Conway result for nonlocally compact spaces. We do not consider these extensions here.

1. The categories $B_{1}$ and MIXTOP. We consider the categories:

$\mathrm{BAN}_{1}$-whose objects are complex Banach spaces and whose morphisms are linear contractions;

MIXTOP-the objects of MIXTOP are triples $(E,\|\|, \tau)$ where $E$ is a complex vector space, \|\| is a norm on $E$ and $\tau$ is a locally convex topology on $E$ so that $B_{\|\|}$, the unit ball of $(E,\|\|)$, is $\tau$-complete and bounded. The MIXTOP-morphisms from $(E,\|\|, \tau)$ into $\left(F,\|\|_{1}, \tau_{1}\right)$ are the line ar norm contractions from $E$ into $F$ whose restrictions to $B\|\|$ are $\tau-\tau{ }_{1}$ continuous.

As is well known, BAN ${ }_{1}$ and MIXTOP are complete, that is, they possess products and, more generally, projective limits. We recall briefly how products are constructed. Let $\left\{\left(E_{\alpha},\|\|_{\alpha}\right)\right\}_{\alpha \in A}$ be a family of objects in $B N_{1}$. Define

$$
E:=\left\{\left(x_{a}\right) \in \prod E_{a}: \sup _{\alpha \in A}\left\|x_{\alpha}\right\|_{\alpha}<\infty\right\} .
$$

Then $E$, with the norm $\left(x_{a}\right) \mapsto \sup _{a}\left\|x_{a}\right\|_{a}$, is the $\mathrm{BAN}_{1}$-product of $\left\{\left(E_{a},\|\|_{\alpha}\right)\right\}$. If $\left\{\left(E_{a},\|\|_{a}, \tau_{a}\right)\right\}_{a \in A}$ is a family of objects in MIXTOP, then we construct a normed space $(E,\|\|)$ as above and let $\tau$ be the locally convex topology induced on $E \subseteq \Pi_{\alpha \in A} E_{a}$ by the product topology on $\Pi E_{\alpha}$. Then $(E,\|\|, \tau)$ is an object of MIXTOP and is the MIXTOP product of the family $\left\{\left(E_{a},\|\|_{a}, \tau_{a}\right)\right\}_{\text {. }}$ The projective limits of projective systems in $\mathrm{BAN}_{1}$ and MIXTOP are constructed as subspaces of products as usual.

If $\left\{\left(E_{\alpha},\|\|_{\alpha}\right)\right\}_{\alpha \in A}$ is a family of Banach spaces, we denote its $\mathrm{BAN}_{1}$ product by $\mathrm{B} \mathrm{II}_{a \in A} E_{\alpha}$. We can regard each $\left(E_{\alpha},\|\|_{\alpha}\right)$ as an object of MIXTOP by taking $\tau_{a}$ to be the locally convex topology induced by \|\|$_{a}$. We denote by MT $\Pi E_{a}$ the MIXTOP-product of this family.

2. The functor $\gamma$. If $(E,\|\|, \tau)$ is an object of MIXTOP, we define $\gamma[\|\|, \tau]$ to be the finest locally convex topology on $E$ which agrees with $\tau$ on $B_{\|} \|$. The correspondence which associates to each $(E,\|\|, \tau)$ the locally convex space $(E, \gamma[\|\|, \tau])$ and leaves morphisms unchanged is a functor $\gamma$ from MIXTOP into the category of locally convex spaces. 
3. Sums in $B A N_{1}$. The category $B A N_{1}$ is also cocomplete-in particular, it possesses sums. If $\left\{\left(E_{a},\|\|_{a}\right)\right\}_{a \in A}$ is a family of objects in $B A N_{1}$, the space

$$
E_{0}:=\left\{\left(x_{\alpha}\right) \in \prod E_{a}: \sum_{a \in A}\left\|x_{a}\right\|_{\alpha}<\infty\right\}
$$

with the norm \|\|$_{0}:\left(x_{\alpha}\right) \mapsto \Sigma_{\alpha \in A}\left\|x_{\alpha}\right\|_{\alpha}$ is a Banach space. It is the $B A N_{1}$ sum of $\left\{\left(E_{a},\|\|_{\alpha}\right)\right\}$ and we denote it by B $\Sigma_{a \in A} E_{a}$.

4. Duality theory for MIXTOP objects. If $(E,\|\|, \tau)$ is an object of MIXTOP, we define the $\gamma$-dual space of $E$ to be $(E, \gamma[\|\|, \tau])^{\prime}$ with the strong topology. We write $E_{\gamma}^{\prime}$ for this space. Then $E_{\gamma}^{\prime}$ is the norm closure of $(E, \tau)^{\prime}$ in the Banach space $(E,\|\|)^{\prime}[5]$ and so is a Banach space.

5. Proposition. Let $\left\{\left(E_{\alpha},\|\|_{\alpha}\right)\right\}_{\alpha \in A}$ be a family in $\mathrm{BAN}_{1}$. Then

(i) the mapping $\left(\left(x_{\alpha}\right),\left(f_{\alpha}\right)\right) \mapsto \Sigma_{\alpha \in A} f_{\alpha}\left(x_{\alpha}\right)$ makes ( $\left.B \Pi E_{\alpha}, B \Sigma E_{\alpha}^{\prime}\right)$ a dual pair;

(ii) under this duality, $\mathrm{B} \Sigma E_{a}^{\prime}$, with its Banach space structure, is identifiable with the $\gamma$-dual of MT $\Pi E_{a}$;

(iii) a subset $C$ of $\mathrm{B} \Sigma E_{a}^{\prime}$ is $\gamma[\|\|, \tau]$-equicontinuous if and only if it is norm bounded and, for each $\epsilon>0$, there is a finite subset $J$ of $A$ so that $\Sigma_{a \in A \backslash J}\left\|f_{a}\right\| \leq \epsilon$ for each $\left(f_{a}\right) \in C$.

Proof. (i) is a routine calculation and (ii) follows from the characterization of $E_{y}^{\prime}$ given above and the fact that the locally convex direct sum $\bigoplus\left(E_{\alpha}, \tau_{\alpha}\right)^{\prime}$ can be identified with the dual of $\left(\Pi E_{\alpha}, \Pi \tau_{\alpha}\right)$ under the duality given formally as in the statement of the proposition. (iii) follows from the characterization of the equicontinuous sets of $\bigoplus E_{\alpha}^{\prime}$ and the following

6. Lemma $([7,2.2])$. Let $(E,\|\|, \tau)$ be an object of MIXTOP. Then a subset $C$ of $E_{\gamma}^{\prime}$ is $\gamma$-equicontinuous if and only if the following condition holds:

For every $\epsilon>0$, there is a r-equicontinuous subset $C_{1}$ of $(E, \tau)^{\prime}$ so that $C \subseteq \epsilon B+C_{1}$ where $B$ is the unit ball of $(E,\|\|)^{\prime}$.

7. Proposition. Let $\left\{\left(E_{a},\|\|_{a}\right)\right\}_{a \in A}$ be as in 5. Then on B $\Pi E_{a}, \gamma[\|\|, \tau]$ is the Mackey topology for the duality (B ПI $E_{a}, \mathrm{~B} \Sigma E_{a}^{\prime}$ ).

Proof. By 5(iii) it is sufficient to show that if $C$ is a $\sigma\left(\mathrm{B} \Sigma E_{\alpha}^{\prime}, \mathrm{B} \Pi E_{\alpha}\right)$ compact subset of $\mathrm{B} \Sigma E_{\alpha}^{\prime}$, then $C$ satisfies the condition given there. We show first that the support $A_{1}$ of $C$ is countable $\left(A_{1}\right.$ is the set of $\beta \in A$ for which an $\left(f_{a}\right) \in C$ exists with $\left.f_{\beta} \neq 0\right)$. For each $\beta \in A_{1}$ we choose an $x_{\beta} \in E_{\beta}$ so that $\left\|x_{\beta}\right\|=1$ and $f_{\beta}\left(x_{\beta}\right) \neq 0$ for some $\left(f_{\alpha}\right) \in C$. Then the mapping $\left(f_{\alpha}\right) \mapsto\left(f_{\alpha}\left(x_{\alpha}\right)\right)$ from $B \Sigma E_{\alpha}^{\prime}$ into $\ell^{1}(A)$ is $\sigma\left(B \Sigma E_{\alpha}^{\prime}\right.$, B II $\left.E_{\alpha}\right)$ $\sigma\left(l^{1}\left(A_{1}\right), l^{\infty}\left(A_{1}\right)\right)$ continuous. The image of $C$ under this mapping is weakly compact and so has countable support. But, by construction, the support of this set is $A_{1}$. 
Thus we can consider the case where the indexing set is $\mathbf{N}$. If $C$ does not satisfy the condition of 5 (iii) then there is a strictly increasing sequence of positive integers $\left(n_{k}\right)$ and a sequence $\left(f^{(k)}\right)$ in $C$ so that

$$
\sum_{n=n_{k}+1}^{n_{k+1}}\left\|f_{n}^{(k)}\right\| \geq \epsilon \text { and } \sum_{n>n_{k+1}}\left\|f_{n}^{(k)}\right\| \leq \epsilon / 3
$$

for some positive $\epsilon$.

There is an $x_{n} \in E_{n}$ so that $\left\|x_{n}\right\|=1$ and $f_{n}^{(k)}\left(x_{n}\right) \geq(2 / 3)\left\|f_{n}^{(k)}\right\|$ $\left(n_{k}<n \leq n_{k+1}\right)$. Let $x$ denote the element $\left(x_{n}\right)$ of $\mathrm{B} \Pi E_{n}$. Then

$$
\sum_{n>n_{k}}\left|f_{n}^{(k)}\left(x_{n}\right)\right|=\sum_{n=n_{k}+1}^{n+1}\left|f_{n}^{(k)}\left(x_{n}\right)\right|+\sum_{n>n_{k+1}}\left|f_{n}^{(k)}\left(x_{n}\right)\right| \geq \epsilon / 3 .
$$

Hence the set $\left\{\left(f_{n}\left(x_{n}\right)\right): f \in C\right\}$ is not weakly compact in $\ell^{1}(\mathrm{~N})$ by Schur's theorem (Trèves $[14$, p. 450]).

8. Partitions of unity in MIXTOP. Let $\left\{\pi_{\beta a}: E_{\beta} \rightarrow E_{\alpha} ; \alpha, \beta \in A\right\}$ be a projective system in $\mathrm{BAN}_{1}, E$ its projective limit and $\pi_{\alpha}: E \rightarrow E_{\alpha}$ the natural projection. We write $\mathrm{B} \operatorname{Proj}\left(E_{\alpha}\right)$ for $E$ with its natural Banach space structure; MT Proj $\left(E_{\alpha}\right)$ for $E$ with its MIXTOP structure as the projective limit in the category MIXTOP.

A MIXTOP partition of unity is a family $\left\{T_{a}\right\}$ of $\mathrm{BAN}_{1}$-morphisms where $T_{a}$ maps $E_{\alpha}$ into $E$ and

(i) for each $\beta \in A,\left\{\alpha \in A: \pi_{\beta} \circ T_{\alpha} \neq 0\right\}$ is finite;

(ii) for each finite $J \subseteq A$, $\left\|\Sigma_{a \in J} T_{a} \circ \pi_{a}\right\| \leq 1$;

(iii) for each $x \in E, \Sigma_{a \in A} T_{\alpha} \circ \pi_{a}(x)=x$ (convergence in $\tau$ ).

9. Example. Let $S$ be a locally compact, paracompact space and suppose that $\left\{\phi_{a}: a \in A\right\}$ is a partition of unity on $S$ (Bourbaki [1, IX. 4.3]). Let $K_{a}$ : = supp $\phi_{a}$. Then if $C(S)$ denotes the Banach space of all bounded, continuous, complex-valued functions on $S$ with the supremum norm \|\|$_{\infty}$ and if $\tau_{c}$ denotes the topology of compact convergence, then $\left(C(S),\|\|_{\infty}, \tau_{c}\right)$ is an object of MIXTOP and $\gamma\left(C(S),\|\|_{\infty}, \tau_{c}\right)$ is $(C(S), \beta)$ (Buck [2], Cooper [5]). $\left(C(S),\|\|, \tau_{c}\right)$ is easily identifiable with the MIXTOP projective limit of the system of Banach spaces $\left\{C\left(K_{\alpha}\right): a \in A\right\}$ and if we define the mappings $T_{a}$ : $x \mapsto\left(x \phi_{a}\right)^{\wedge}$ from $C\left(K_{a}\right)$ into $C(S)$ where $\left(x \phi_{a}\right)^{\wedge}$ denotes the extension of $x \phi_{a}$ to a function on $S$ obtained by setting it equal to zero off $K_{a}$, then $\left\{T_{a}\right\}$ is a MIXTOP partition of unity.

10. Proposition. With the notation of $8, \gamma\left(\mathrm{MT} \operatorname{Proj}\left(E_{\alpha}\right)\right)$ is a quotient space of $\gamma\left(\mathrm{MT} \Pi E_{\alpha}\right)$.

Proof (cf. Keim [9]). The natural mapping $x \mapsto\left(\pi_{\alpha}(x)\right)_{\alpha \in A}$ embeds $E$ as a subspace of $\mathrm{B} \Pi E_{\alpha}$. On the other hand, the mapping $T:\left(x_{\alpha}\right) \rightarrow \Sigma_{\alpha \in A} T_{\alpha} x_{\alpha}$ from $\mathrm{B} \Pi E_{\alpha}$ into $E$ is a MIXTOP morphism and is a left inverse for this embedding. 
11. Corollary. The locally convex space $\gamma\left(\mathrm{MT} \operatorname{Proj}\left(E_{\alpha}\right)\right)$ is a Mackey space, that is, its topology is the Mackey topology.

12. Corollary (LeCam, Conway). If $S$ is a locally compact, paracompact space, then $(C(S), \beta)$ is a Mackey space.

13. Definition. A locally convex space $E$ has the Banach-Steinhaus property if, for every pointwise convergent sequence of continuous linear operators from $E$ into a locally convex space $F$, the limit operator is continuous. In this definition, it is clearly sufficient to assume that $F$ is a Banach space. It can be easily seen that this property is inherited by quotient spaces.

14. Remark. Let $\left\{\left(E_{\alpha},\|\|_{\alpha}\right)\right\}_{a \in A}$ be as in 5. We can regard each $E_{\alpha}$ as a subspace of $\mathrm{B} \Pi_{\alpha \in A} E_{\alpha}$ or of $\mathrm{MT} \Pi_{a \in A} E_{a}$ in a natural way. If $T$ is a continuous linear operator from MT $\Pi E_{\alpha}$ into a Banach space $F$ and $T_{\alpha}:\left.T\right|_{E_{\alpha}}$, then $\|T\|=\Sigma_{a \epsilon A}\left\|T_{a}\right\|$. Conversely, if $\left\{T_{a}\right\}_{\alpha \epsilon A}$ is a family of continuous linear operators where $T_{\alpha}$ maps $E_{a}$ into $F$, then there is a continuous linear operator $T$ from MT II $E_{a}$ into $F$ so that $\left.T\right|_{E_{\alpha}}=T_{\alpha}$ for each $\alpha \in A$ if and only if $\Sigma_{a \in A}\left\|T_{a}\right\|<\infty$, and this $T$ is then unique. (Note that the above considerations are only valid because we restrict our attention to the operators from $\mathrm{B} \Pi E_{\alpha}$ into $F$ which are continuous for the mixed structure on $\mathrm{B} \Pi E_{\alpha}$. They do not, of course, hold for general continuous linear operators from В П $E_{a}$ into $F$.)

15. Proposition. Let $\left\{\left(E_{\alpha},\|\|_{\alpha}\right)\right\}_{\alpha \in A}$ be as in 5. Then $\gamma\left(\mathrm{MT} \Pi E_{\alpha}\right)$ has the Banach-Steinhaus property.

Proof. Let $\left(T_{n}\right)$ be a pointwise convergent sequence of continuous linear operators from MT $\Pi E_{\alpha}$ into a Banach space $F$ and denote $\left.T_{n}\right|_{E_{\alpha}}$ by $T_{n}^{\alpha}$. Then the sequence $\left(T_{n}^{a}\right)_{n \in \mathrm{N}}$ is pointwise convergent to a continuous linear operator $T_{\alpha}$ from $E_{\alpha}$ into $F$ for each $\alpha \in A$. By the principle of uniform boundedness, $\sup \left\{\left\|T_{n}\right\|: n \in \mathrm{N}\right\}<\infty$ and so $\sup \left\{\Sigma_{a \in A}\left\|T_{n}^{a}\right\|: n \in \mathbf{N}\right\}<\infty$. From this it follows that $\Sigma_{a \in A}\left\|T_{a}\right\|<\infty$, and so there is a continuous linear operator $T$ from MT $\Pi E_{\alpha}$ into $F$ so that $\left.T\right|_{E_{\alpha}}=T_{\alpha}$ for each $a \in A$. A simple $\epsilon / 3$ argument shows that $T$ is the pointwise limit of the sequence $\left(T_{n}\right)$.

16. Corollary. With the notation of $8, \gamma\left(\mathrm{MT}\right.$ Proj $\left.\left(E_{a}\right)\right)$ has the BanachSteinhaus property.

17. Corollary. If $S$ is a locally compact, paracompact space, then $(C(S), \beta)$ has the Banach-Steinhaus property.

18. Bibliographical remarks. The categories $\mathrm{BAN}_{1}$ and MIXTOP are discussed by Semadeni [11]. The functor $\gamma$ was introduced by Wiweger. We refer to [5] for the properties of $\gamma$ required and for a bibliography of the original papers. Result 12 was published by LeCam [10] (for $\sigma$-compact $S$ ) and 
Conway [4]. For generalizations to nonlocally compact spaces, we refer to Fremlin, et al. [8], Sentilles [12] and Wheeler [15]. Partitions of unity for the category of locally convex spaces were introduced by De Wilde [16] (see also Keim [9]). For result 17, compare Theorem 5.1 of Collins and Dorroh [3].

\section{REFERENCES}

1. N. Bourbaki, Elements of mathematics. General topology. Part 2, Hermann, Paris; Addison-Wesley, Reading, Mass., 1966. MR 34 \#5044b; erratum, 40, p. 1704.

2. R. C. Buck, Bounded continuous functions on a locally compact space, Michigan Math. J. 5 (1958), 95-104. MR 21 \#3350.

3. H. S. Collins and J. R. Dorroh, Remarks on certain function spaces, Math. Ann. 176 (1968), 157-168. MR 36 \#5694.

4. J. B. Conway, The strict topology and compactness in the space of measures. II, Trans. Amer. Math. Soc. 126 (1967), 474-486. MR 34 \#6503.

5. J. B. Cooper, The strict topology and spaces with mixed topologies, Proc. Amer. Math. Soc. 30 (1971), 583-592. MR 44 \#2013.

6. - Topologies sur l'espace des opérateurs dans l'espace hilbertien, C. R. Acad. Sci. Paris Sér. A-B 276 (1973), A 1509-A1511. MR 47 \#7519.

7. - Compactologies and spaces of bounded continuous functions, Institutsbericht-Hochschule Linz, August 1974.

8. D. H. Fremlin, D. J. H. Garling and R. G. Haydon, Bounded measures on topological spaces, Proc. London Math. Soc. (3) 25 (1972), $115-136$.

9. D. Keim, Induktive und projektive Limiten mit Zerlegung der Einheit, Manuscripta Math. 10 (1973), 191-195.

10. L. LeCam, Convergence in distribution of stochastic processes, Univ. Calif. Publ. Statist. 2 (1957), 207-236. MR 19, 128.

11. Z. Semadeni, Projectivity, injectivity and duality, Rozprawy Mat. 35 (1963), 47 pp. MR 27 \#4776.

12. F. D. Sentilles, Bounded continuous functions on a completely regular space, Trans. Amer. Math. Soc. 168 (1972), 311-336. MR 45 \#4133.

13. K. D. Stroyan, A characterization of the Mackey uniformity $m\left(L^{\infty}, L^{1}\right)$ for finite measures, Pacific J. Math. (to appear).

14. F. Trèves, Topological vector spaces, distributions and kernels, Academic Press, New York and London, 1967. MR 37 \#26.

15. R. F. Wheeler, The strict topology, separable measures and paracompactness, Pacific J. Math. 47 (1973), 287-302.

16. M. De Wilde, Inductive limits and partitions of unity, Manuscripta Math. 5 (1971), 45-58. MR $45 \# 4092$. 